\title{
An Approached Solution of Wave Equation with Cubic Damping by Homotopy Perturbation Method (HPM), Regular Pertubation Method (RPM) and Adomian Decomposition Method (ADM)
}

\author{
Moussa BAGAYOGO ${ }^{1}$, Youssouf PARE $^{1} \&$ Youssouf MINOUNGOU ${ }^{1}$ \\ ${ }^{1}$ Département de mathématiques, Université Ouaga I Professeur Joseph Ki-Zerbo, Burkina Faso \\ Correspondence: Youssouf PARE, Département de mathématiques, Université Ouaga I Professeur Joseph Ki-Zerbo, Burk- \\ ina Faso.
}

Received: January 2, 2018 Accepted: January 22, 2018 Online Published: March 29, 2018

doi:10.5539/jmr.v10n2p166 URL: https://doi.org/10.5539/jmr.v10n2p166

\begin{abstract}
In this study, we consider the wave equation with cubic damping with its initial conditions. Homotopy Perturbation Method (HPM), Regular Pertubation Method (RPM) and Adomian decomposition Method (ADM) are applied to this equation. Then, the solution yielding the given initial conditions is gained. Finally, the solutions obtained by each method are compared.
\end{abstract}

Keywords: Wave equation, cubic damping, Homotopy Perturbation Method (HPM), Regular Pertubation Method (RPM), Adomian decomposition Method (ADM)

\section{Introduction}

Over the last decades, several analytical/approximate methods have been developed to solve ordinary and partial differential equations. Some of these techniques include Homotopy Perturbation Method (He, J. H., 1999; He, J. H., 2000; He, J. H., 2003; He, J. H., 2004; Gupta, S. \& et al., 2013), Regular Pertubation Method (Ghazanfari, B., 2011) and Adomian decomposition Method (ABBAOUI, K., 1995; ABBAOUI, K. \& CHERRUAULT, Y., 1994; ABBAOUI, K. \& CHERRUAULT, Y., 1999; NGARHASTA, N. \& et al., 2002; Oke, M. O., 2015; Ghoreishi, M. \& et al, 2010), etc.

Linear and nonlinear phenomena are of fundamental importance in various fields of science and engineering. Most models of real-life problems, however, are still very difficult to solve. Therefore, approximate analytical solutions such as HPM, RPM and ADM were introduced, which are effective and convenient for both linear and nonlinear equations.

In this paper, we consider the following nonlinearly damped wave equation

$$
\left\{\begin{array}{c}
\frac{\partial^{2} u}{\partial t^{2}}-\frac{\partial^{2} u}{\partial x^{2}}=-\varepsilon\left(\frac{\partial u}{\partial t}\right)^{3}, t \geq 0, x \in \mathbb{R} \\
u(0, x)=\cos x, \frac{\partial u}{\partial t}(0, x)=0
\end{array}\right.
$$

Where $\varepsilon$ is perturbation parameter which, $u(t, x)$ is some physical quantity, $x$ the space variable and $t$ stands for time.

These types of equations are of considerable significance in various fields of applied sciences, mathematical physics, nonlinear hydrodynamics, engineering physics, biophysics, human movement sciences, astrophysics and plasma physics. The paper is organised as follows : in section 1, we start with the solving (1) by HPM. In Section 2 and section 3 , we construct the solution of (1) respectively by RPM (He, J. H., 2004) and ADM. Section 4 contains the comparison of the solutions obtained by the different methods.

\section{Homotopy Perturbation Method}

\subsection{Basic Idea of the Homotopy Perturbation Method}

To illustrate the basic idea of HPM, consider the following nonlinear differential equation

$$
A(u)=f(r), \quad r \in \Omega
$$

with boundary conditions

$$
B\left(u, \frac{\partial u}{\partial n}\right)=0, \quad r \in \Gamma
$$


where $A$ is a general differential operator, $B$ is a boundary operator, $f(r)$ is a known analytic function, and $\Gamma$ is the boundary of the domain $\Omega$. Generally speaking, the operator $A$ can be decomposed into two parts $L$ and $N$, where $L$ is a linear and $N$ is a nonlinear operator. Equation (2), therefore, can be rewritten as follows :

$$
L(u)+N(u)-f(r)=0
$$

We construct a homotopy $v(r, p): \Omega \times[0,1] \rightarrow \mathbb{R}$, that satisfies

$$
H(v, p)=(1-p)\left[L(v)-L\left(u_{0}\right)\right]+p[A(v)-f(r)]=0, p \in[0,1], r \in \Omega
$$

or, equivalently,

$$
H(v, p)=L(v)-L\left(u_{0}\right)+p L\left(u_{0}\right)+p[N(v)-f(r)]=0
$$

where $u_{0}$ is an initial approximation to the solution of Equation (2). In this method, we use the homotopy parameter $p$ to expand $v$ as a power series

$$
v=v_{0}+p v_{1}+p^{2} v_{2}+p^{3} v_{3}+p^{4} v_{4}+p^{5} v_{5}+\cdots
$$

The approximate solution can be obtained by setting $p=1$,

$$
u=\lim p \rightarrow 1 v=v_{0}+v_{1}+v_{2}+\cdots
$$

The convergence of the series of (4) has been proved in (He, J. H., 1999; He, J. H., 2000).

\subsection{Application of HPM Wave Equation with Cubic Damping}

According to the HPM (He, J. H., 1999; He, J. H., 2003; He, J. H., 2004; Gupta, S. \& et al., 2013), we can construct the homotopie $H(v, p)$ for equation (1) which satisfies :

$$
H(v, p)=(1-p)\left[\frac{\partial^{2} v}{\partial t^{2}}-\frac{\partial^{2} u_{0}}{\partial t^{2}}\right]+p\left[\frac{\partial^{2} v}{\partial t^{2}}-\frac{\partial^{2} v}{\partial x^{2}}+\varepsilon\left(\frac{\partial v}{\partial t}\right)^{3}\right]
$$

As $H(v, p)=0$, then we have :

$$
\frac{\partial^{2} v}{\partial t^{2}}-\frac{\partial^{2} u_{0}}{\partial t^{2}}+p \frac{\partial^{2} u_{0}}{\partial t^{2}}-p \frac{\partial^{2} v}{\partial x^{2}}+p \varepsilon\left(\frac{\partial v}{\partial t}\right)^{3}=0
$$

Let as choose the initial approximation as $u_{0}=\cos x$, thus $\frac{\partial^{2} u_{0}}{\partial t^{2}}=\frac{\partial u_{0}}{\partial t}=0$

We have,

$$
\frac{\partial^{2} v}{\partial t^{2}}-p \frac{\partial^{2} v}{\partial x^{2}}+p \varepsilon\left(\frac{\partial v}{\partial t}\right)^{3}=0
$$

Assume the solution of (1) to be in the form :

$$
v=v_{0}+p v_{1}+p^{2} v_{2}+p^{3} v_{3}+p^{4} v_{4}+p^{5} v_{5}+\cdots
$$

Substituting (6) into (5) and equating the coefficients of like powers $p$, we get the following set of differential equations :

$$
\begin{gathered}
p^{0}:\left\{\begin{array}{c}
\frac{\partial^{2} v_{0}}{\partial t^{2}}-\frac{\partial^{2} u_{0}}{\partial t^{2}}=0 \\
v_{0}(0, x)=\cos x, \frac{\partial v_{0}}{\partial t}(0, x)=0
\end{array}\right. \\
p^{1}:\left\{\begin{array}{c}
\frac{\partial^{2} v_{1}}{\partial t^{2}}-\frac{\partial^{2} v_{0}}{\partial x^{2}}+3 \varepsilon\left(\frac{\partial v_{0}}{\partial t}\right)^{2}\left(\frac{\partial^{2} v_{1}}{\partial t}\right)=0 \\
v_{1}(0, x)=0, \frac{\partial v_{1}}{\partial t}(0, x)=0
\end{array}\right. \\
p^{2}:\left\{\begin{array}{c}
\frac{\partial^{2} v_{2}}{\partial t^{2}}-\frac{\partial^{2} v_{1}}{\partial x^{2}}+3 \varepsilon\left(\frac{\partial v_{0}}{\partial t}\right)^{2}\left(\frac{\partial v_{2}}{\partial t}\right)+3 \varepsilon\left(\frac{\partial v_{0}}{\partial t}\right)\left(\frac{\partial v_{1}}{\partial t}\right)^{2}=0 \\
v_{2}(0, x)=0, \frac{\partial v_{2}}{\partial t}(0, x)=0
\end{array}\right.
\end{gathered}
$$




$$
p^{3}:\left\{\begin{array}{c}
\frac{\partial^{2} v_{3}}{\partial t^{2}}-\frac{\partial^{2} v_{2}}{\partial x^{2}}+3 \varepsilon\left(\frac{\partial v_{0}}{\partial t}\right)^{2}\left(\frac{\partial v_{3}}{\partial t}\right)+6 \varepsilon\left(\frac{\partial v_{0}}{\partial t} \frac{\partial v_{1}}{\partial t} \frac{\partial v_{2}}{\partial t}\right)+\varepsilon\left(\frac{\partial v_{1}}{\partial t}\right)^{3}=0 \\
v_{3}(0, x)=0, \frac{\partial v_{3}}{\partial t}(0, x)=0
\end{array}\right.
$$

From the above equations, we can obtain

$$
\begin{aligned}
& v_{0}(t, x)=\cos x \\
& v_{1}(t, x)=-\frac{1}{2} t^{2} \cos x \\
& v_{2}(t, x)=\frac{1}{24} t^{4} \cos x \\
& v_{3}(t, x)=-\frac{1}{720} t^{6} \cos x+\frac{\varepsilon}{20} t^{5} \cos ^{3} x \\
& v_{4}(t, x)=\frac{\varepsilon}{140} t^{7} \cos x \sin ^{2} x-\frac{13 \varepsilon}{840} t^{7} \cos ^{3} x+\frac{1}{40320} t^{8} \cos x \\
& v_{5}(t, x)=-\frac{1}{3628800} t^{10} \cos x-\frac{\varepsilon}{504} t^{9} \cos x \sin ^{2} x+\frac{71 \varepsilon}{30240} t^{9} \cos ^{3} x-\frac{3 \varepsilon^{2}}{224} t^{8} \cos ^{5} x
\end{aligned}
$$

In principle, it is possible to calculate more components in the expansion series to enhance the approximation. Therefore, we get the tenth-order approximation,

$$
\begin{aligned}
& u(t, x)=\left[\cos x\left(1-\frac{t^{2}}{2 !}+\frac{t^{4}}{4 !}-\frac{t^{6}}{6 !}+\frac{t^{8}}{8 !}-\frac{t^{10}}{10 !}+\frac{t^{12}}{12 !}-\frac{t^{14}}{14 !}+\frac{t^{16}}{16 !}-\frac{t^{18}}{18 !}+\frac{t^{20}}{20 !}\right)\right]+ \\
& \varepsilon\left[\operatorname { c o s } ^ { 3 } x \left(\frac{t^{5}}{20}-\frac{13 t^{7}}{840}+\frac{71 t^{9}}{30240}-\frac{491 t^{11}}{3326400}+\frac{13711 t^{13}}{1037836800}-\frac{28607 t^{15}}{43589145600}\right.\right. \\
& +\frac{66811 t^{17}}{2694601728000}-\frac{7198319 t^{19}}{10137091700736000}+\frac{t^{21}}{13502619648000} \\
& \left.-\frac{61 t^{23}}{47826278793216000}+\frac{547 t^{25}}{28695767275929600000}\right) \\
& +\cos x \sin ^{2} x\left(\frac{t^{7}}{140}-\frac{t^{9}}{504}+\frac{47 t^{11}}{184800}-\frac{739 t^{13}}{43243200}+\frac{34403 t^{15}}{36324288000}-\frac{727 t^{17}}{18712512000}\right. \\
& \left.\left.+\frac{188947 t^{19}}{153592298496000}-\frac{t^{21}}{4725916876800}+\frac{13 t^{23}}{3416162770944000}-\frac{41 t^{25}}{717394181898240000}\right)\right] \\
& +\varepsilon^{2}\left[\operatorname { c o s } ^ { 5 } x \left(-\frac{3 t^{8}}{224}+\frac{439 t^{10}}{100800}-\frac{23801 t^{12}}{13305600}+\frac{743201 t^{14}}{2421619200}-\frac{38239 t^{16}}{931392000}+\frac{78121361 t^{18}}{22230464256000}\right.\right. \\
& \left.-\frac{1903 t^{20}}{482236416000}+\frac{5921 t^{22}}{31191051386880}-\frac{297239 t^{24}}{31304473391923200}\right) \\
& +\cos ^{3} x \sin ^{2} x\left(-\frac{13 t^{10}}{2800}+\frac{3397 t^{12}}{1663200}-\frac{352421 t^{14}}{605404800}+\frac{83371 t^{16}}{825552000}-\frac{26118227 t^{18}}{2223046425600}\right. \\
& \left.+\frac{1247 t^{20}}{42195686400}-\frac{1191041 t^{22}}{779776284672000}+\frac{11675131 t^{24}}{215218254569472000}+\frac{12517 t^{25}}{1016715110400000}\right) \\
& +\cos x \sin ^{4} x\left(-\frac{13 t^{12}}{61600}+\frac{1663 t^{14}}{20180160}-\frac{16447 t^{16}}{864864000}+\frac{573889 t^{18}}{205837632000}-\frac{2903 t^{20}}{281304576000}\right. \\
& \left.\left.+\frac{12517 t^{23}}{20334302208000}-\frac{1191041 t^{24}}{71739418189824000}-\frac{162721 t^{25}}{12200581324800000}\right)\right]+ \\
& \varepsilon^{3}\left[\operatorname { c o s } ^ { 7 } x \left(\frac{57 t^{11}}{12320}-\frac{1319 t^{13}}{443520}+\frac{54919 t^{14}}{47297250}+\frac{83, t^{15}}{470400}-\frac{54919 t^{16}}{1621620000}-\frac{227253749 t^{17}}{823350528000}\right.\right.
\end{aligned}
$$




$$
\begin{aligned}
& \left.+\frac{1901771 t^{19}}{1969132032000}-\frac{37259 t^{21}}{170031960000}+\frac{215576267 t^{23}}{7608726171648000}\right)+\cos ^{5} x \sin ^{2} x\left(\frac{839 t^{13}}{320320}\right. \\
& \left.-\frac{165827 t^{15}}{84084000}+\frac{54919 t^{16}}{270270000}+\frac{76842539 t^{17}}{137225088000}-\frac{947 t^{19}}{202585600}+\frac{5031053 t^{21}}{7254696960000}-\frac{34493909 t^{23}}{333716060160000}\right) \\
& +\cos ^{3} x \sin ^{4} x\left(+\frac{193 t^{15}}{600600}-\frac{65171 t^{17}}{285885600}+\frac{129 t^{19}}{40517120}-\frac{5819 t^{21}}{12155136000}+\frac{277663 t^{23}}{4359166035840}\right)+ \\
& \left.\cos x \sin ^{6} x\left(\frac{193 t^{17}}{27227200}-\frac{t^{19}}{6077568}+\frac{193 t^{21}}{3646540800}-\frac{35353 t^{23}}{4612874112000}\right)\right] \\
& +\varepsilon^{4}\left[\cos ^{9} x\left(-\frac{1097 t^{14}}{407680}+\frac{557813 t^{16}}{358758400}-\frac{2383 t^{18}}{83166720}+\frac{1830301 t^{20}}{210689024000}-\frac{12469598887 t^{22}}{7884404656128000}\right)+\right. \\
& \cos ^{7} x \sin ^{2} x\left(-\frac{76059 t^{16}}{44844800}+\frac{29 t^{18}}{495040}-\frac{183721 t^{20}}{8103424000}+\frac{2080131 t^{22}}{331082752000}\right) \\
& +\cos ^{5} x \sin ^{4} x\left(-\frac{9 t^{18}}{247520}+\frac{6543 t^{20}}{526722560}-\frac{103197433 t^{22}}{24334582272000}\right)+\cos ^{3} x \sin ^{6} x\left(-\frac{9 t^{20}}{4702880}\right. \\
& \left.\left.+\frac{647 t^{22}}{869092224}\right)-\cos x \sin ^{8} x \frac{9 t^{22}}{362121760}\right] \\
& +\varepsilon^{5}\left[\cos ^{11} x\left(\frac{27 t^{17}}{198016}-\frac{11899 t^{19}}{263361280}+\frac{440053 t^{21}}{22122347520}\right)+\cos ^{9} x \sin ^{2} x\left(\frac{10671 t^{19}}{131680640}-\frac{156787 t^{21}}{3456616800}\right)\right. \\
& \left.+\cos ^{7} x \sin ^{4} x \frac{21393 t^{21}}{1152205600}\right]+\varepsilon^{6}\left[-\cos ^{13} x \frac{297 t^{20}}{4426240}\right]
\end{aligned}
$$

This solution can be written in the form :

$$
\begin{aligned}
u(t, x) \simeq & \cos t \cos x+\varepsilon\left[k_{1}(t) \cos ^{3} x+k_{2}(t) \cos x \sin ^{2} x\right] \\
& +\varepsilon^{2}\left[k_{3}(t) \cos ^{5} x+k_{4}(t) \cos ^{3} x \sin ^{2} x+k_{5}(t) \cos x \sin ^{4} x\right] \\
& +\varepsilon^{3}\left[k_{6}(t) \cos ^{7} x+k_{7}(t) \cos ^{5} x \sin ^{2} x+k_{8}(t) \cos ^{3} x \sin ^{4} x+k_{9}(t) \cos x \sin ^{6} x\right] \\
& +\cdots
\end{aligned}
$$

Where

$$
\begin{aligned}
k_{1}(t)= & \frac{t^{5}}{20}-\frac{13 t^{7}}{840}+\frac{71 t^{9}}{30240}-\frac{491 t^{11}}{3326400}+\frac{13711 t^{13}}{1037836800}-\frac{28607 t^{15}}{43589145600}+\frac{66811 t^{17}}{2694601728000} \\
& -\frac{7198319 t^{19}}{10137091700736000}+\frac{t^{21}}{13502619648000}-\frac{61 t^{23}}{47826278793216000}+\frac{547 t^{25}}{28695767275929600000} \\
k_{2}(t)= & \frac{t^{7}}{140}-\frac{t^{9}}{504}+\frac{47 t^{11}}{184800}-\frac{739 t^{13}}{43243200}+\frac{34403 t^{15}}{3632428800}-\frac{727 t^{17}}{18712512000}+\frac{188947 t^{19}}{153592298496000} \\
& -\frac{t^{21}}{4725916876800}+\frac{13 t^{23}}{3416162770944000}-\frac{41 t^{25}}{717394181898240000} \\
k_{3}(t)= & -\frac{3 t^{8}}{224}+\frac{439 t^{10}}{100800}-\frac{23801 t^{12}}{13305600}+\frac{743201 t^{14}}{2421619200}-\frac{38239 t^{16}}{931392000}+\frac{78121361 t^{18}}{22230464256000}-\frac{1903 t^{20}}{482236416000} \\
& +\frac{5921 t^{22}}{31191051386880}-\frac{297239 t^{24}}{31304473391923200} \\
k_{4}(t)= & -\frac{13 t^{10}}{2800}+\frac{3397 t^{12}}{1663200}-\frac{352421 t^{14}}{605404800}+\frac{83371 t^{16}}{825552000}-\frac{26118227 t^{18}}{2223046425600}+\frac{1247 t^{20}}{42195686400} \\
& -\frac{1191041 t^{22}}{779776284672000}+\frac{11675131 t^{24}}{215218254569472000}+\frac{12517 t^{25}}{1016715110400000}
\end{aligned}
$$




$$
\begin{aligned}
k_{5}(t)= & -\frac{13 t^{12}}{61600}+\frac{1663 t^{14}}{20180160}-\frac{16447 t^{16}}{864864000}+\frac{573889 t^{18}}{205837632000}-\frac{2903 t^{20}}{281304576000}+\frac{12517 t^{23}}{20334302208000} \\
& -\frac{1191041 t^{24}}{71739418189824000}-\frac{162721 t^{25}}{12200581324800000} \\
k_{6}(t)= & \frac{57 t^{11}}{12320}-\frac{1319 t^{13}}{443520}+\frac{54919 t^{14}}{47297250}+\frac{83, t^{15}}{470400}-\frac{54919 t^{16}}{1621620000}-\frac{227253749 t^{17}}{823350528000}+\frac{1901771 t^{19}}{1969132032000} \\
& -\frac{37259 t^{21}}{170031960000}+\frac{215576267 t^{23}}{7608726171648000} \\
k_{7}(t)= & \frac{839 t^{13}}{320320}-\frac{165827 t^{15}}{84084000}+\frac{54919 t^{16}}{270270000}+\frac{76842539 t^{17}}{137225088000}-\frac{947 t^{19}}{202585600}+\frac{5031053 t^{21}}{72546969600000} \\
& -\frac{34493909 t^{23}}{333716060160000} \\
k_{8}(t)= & \frac{193 t^{15}}{600600}-\frac{65171 t^{17}}{285885600}+\frac{129 t^{19}}{40517120}-\frac{5819 t^{21}}{12155136000}+\frac{277663 t^{23}}{4359166035840} \\
k_{9}(t)= & \frac{193 t^{17}}{27227200}-\frac{t^{19}}{6077568}+\frac{193 t^{21}}{3646540800}-\frac{35353 t^{23}}{4612874112000}
\end{aligned}
$$

\section{The Regular Perturbation Method}

\subsection{RPM Description}

In order to show, the basic idea of RPM, consider the following differential equation

$$
L_{\varepsilon}\left[u_{\varepsilon}(x)\right]=0, \quad x=\left(x_{1}, x_{2}, \cdots, x_{n}\right) \in \Omega
$$

with boundary conditions

$$
B_{\varepsilon}\left[u_{\varepsilon}(x)\right]=0, \quad x \in \partial \Omega
$$

where $L_{\varepsilon}$ is a genaral differential operator, $B_{\varepsilon}$ is a boundary operator, and $\partial \Omega$

In general, the equations (12)-(13) contain a very small parameter $\varepsilon$. In this method, we use the parameter $u_{\varepsilon}$ as a power series,

$$
u_{\varepsilon}(x)=\sum_{n=0}^{+\infty} \varepsilon^{n} u_{n}(x)
$$

Substituting (14) in (12)-(13), and collecting the coefficient of like powers of $\varepsilon$ yields and equating the coefficient of each power of $\varepsilon$ to zero. We obtain systems of recurrent boundary problems, easy to solve.

The approximate solution is given by

$$
u(x)=u_{0}(x)+\varepsilon u_{1}(x)+\varepsilon^{2} u_{2}(x)+\cdots
$$




\subsection{Application of RPM}

Let us suppose that the solution $u(t, x)$ of the initial value problem (1) has the following form (JAGER, DE. E. M., \& JIANG, FU RU, 1996) :

$$
u(t, x)=\sum_{n=0}^{+\infty} \varepsilon^{n} u_{n}(t, x)
$$

Putting (16) into (1), and collecting equal powers of $\varepsilon$ we obtain a system of recurrent initial value problems

$$
\begin{gathered}
\varepsilon^{0}:\left\{\begin{array}{c}
\frac{\partial^{2} u_{0}}{\partial t^{2}}-\frac{\partial^{2} u_{0}}{\partial x^{2}}=0 \\
u_{0}(0, x)=\cos x, \frac{\partial u_{0}}{\partial t}(0, x)=0
\end{array}\right. \\
\varepsilon^{1:}\left\{\begin{array}{l}
\frac{\partial^{2} u_{1}}{\partial t^{2}}-\frac{\partial^{2} u_{1}}{\partial x^{2}}+\left(\frac{\partial u_{0}}{\partial t}\right)^{3}=0 \\
u_{1}(0, x)=0, \frac{\partial u_{1}}{\partial t}(0, x)=0
\end{array}\right. \\
\varepsilon^{2}:\left\{\begin{array}{c}
\frac{\partial^{2} u_{2}}{\partial t^{2}}-\frac{\partial^{2} u_{2}}{\partial x^{2}}+3\left(\frac{\partial u_{0}}{\partial t}\right)^{2}\left(\frac{\partial u_{1}}{\partial t}\right)=0 \\
u_{2}(0, x)=0, \frac{\partial u_{2}}{\partial t}(0, x)=0
\end{array}\right. \\
\varepsilon^{3}:\left\{\begin{array}{c}
\frac{\partial^{2} u_{3}}{\partial t^{2}}-\frac{\partial^{2} u_{3}}{\partial x^{2}}+3\left(\frac{\partial u_{0}}{\partial t}\right)^{2}\left(\frac{\partial u_{2}}{\partial t}\right)+3\left(\frac{\partial u_{0}}{\partial t}\right)\left(\frac{\partial u_{1}}{\partial t}\right)^{2}=0 \\
u_{3}(0, x)=0, \frac{\partial u_{3}}{\partial t}(0, x)=0
\end{array}\right.
\end{gathered}
$$

To solve $(17,18,19,20)$, we use ADM and we obtain :

$$
\begin{aligned}
u_{0}(t, x)= & \cos t \cos x \\
u_{1}(t, x)= & \cos ^{3} x\left(\frac{43}{108} \sin (3 t)-\frac{75}{4} \sin t\right)+\cos x \sin ^{2} x\left(\frac{99}{2} \sin t-\frac{19}{54} \sin (3 t)\right) \\
u_{2}(t, x)= & \cos ^{5} x\left(-\frac{43}{12} \cos ^{2} t \cos (3 t)-\frac{3914}{625} \cos ^{5} t+\frac{9701909}{40500} \cos ^{3} t+\frac{26472442}{3375} \cos t\right)+ \\
& \cos ^{3} x \sin ^{2} x\left(\frac{19}{6} \cos ^{2} t \cos (3 t)+\frac{23936}{625} \cos ^{5} t-\frac{31274533}{20250} \cos ^{3} t-\frac{216658108}{3375} \cos t\right) \\
& +\cos x \sin ^{4} x\left(-\frac{236}{25} \cos ^{5} t+\frac{200564}{405} \cos ^{3} t+\frac{3396328}{135} \cos t\right) \\
u_{3}(t, x)= & \cos ^{5} x \sin ^{2} x\left(\frac{57}{2} \cos ^{4} t \sin (3 t)+\frac{817}{108} \cos t \cos ^{2}(3 t)+19 \cos ^{3} t \sin t \cos (3 t)\right. \\
& -\frac{947}{2} \cos ^{2} t \cos (3 t)+\frac{71808}{125} \cos ^{6} t \sin t-\frac{31274533}{2250} \cos ^{4} t \sin ^{2} t-\frac{216658108}{1125} \cos ^{2} t \sin t \\
& \left.+\frac{22275}{4} \cos ^{3} t\right)+\cos ^{7} x\left(-\frac{129}{4} \cos ^{4} t \sin (3 t)-\frac{1849}{432} \cos ^{2} \cos ^{2}(3 t)-\frac{43}{2} \cos ^{3} t \sin t \cos (3 t)\right. \\
& +\frac{1075}{8} \cos ^{2} t \cos (3 t)-\frac{11742}{125} \cos ^{6} t \sin t+\frac{9701909}{4500} \cos ^{4} t \sin t+\frac{26472442}{1125} \cos ^{2} t \sin t \\
& \left.-\frac{16875}{16} \cos ^{3} t\right)+\cos ^{3} x \sin ^{4} x\left(-\frac{361}{108} \cos t \cos ^{2}(3 t)+\frac{627}{2} \cos ^{2} t \cos ^{(3 t)-\frac{708}{5} \cos 6} t \sin t+\right. \\
& \left.\frac{200564}{45} \cos ^{4} t \sin t+\frac{3396328}{45} \cos ^{2} t \sin t-\frac{29403}{4} \cos ^{3} t\right)+\cos ^{6} \sin ^{6} x\left(\frac{285 \sin ^{2}(7 t)}{4802}\right.
\end{aligned}
$$




$$
\begin{aligned}
& +\frac{52079 \cos (7 t)}{172872}+\frac{57 \sin (5 t)}{125}+\frac{8683 \cos (5 t)}{9000}-\frac{87932299816 \sin ^{5} t}{16078125}+\frac{169628431490024 \sin ^{3} t}{260465625} \\
& \left.-\frac{2154223646101879 \sin t}{173643750}-\frac{158316 \cos ^{5} t}{125}+\frac{51050891 \cos ^{3} t}{1350}+\frac{1911052939 \cos t}{900}\right)
\end{aligned}
$$

Hence, the approximate solution of (1) is given by :

$$
u(t, x) \simeq u_{0}(t, x)+\varepsilon u_{1}(t, x)+\varepsilon^{2} u_{2}(t, x)+\varepsilon^{2} u_{3}(t, x)
$$

Writting this solution in the form,

$$
\begin{aligned}
u(t, x) \simeq & \cos t \cos x+\varepsilon\left[q_{1}(t) \cos ^{3} x+q_{2}(t) \cos x \sin ^{2} x\right] \\
& +\varepsilon^{2}\left[q_{3}(t) \cos ^{5} x+q_{4}(t) \cos ^{3} x \sin ^{2} x+q_{5}(t) \cos x \sin ^{4} x\right] \\
& +\varepsilon^{3}\left[q_{6}(t) \cos ^{7} x+q_{7}(t) \cos ^{5} x \sin ^{2} x+q_{8}(t) \cos ^{3} x \sin ^{4} x+q_{9}(t) \cos x \sin ^{6} x\right] \\
& +\cdots
\end{aligned}
$$

Where

$$
\begin{aligned}
& q_{1}(t)=\frac{43}{108} \sin (3 t)-\frac{75}{4} \sin t \\
& q_{2}(t)=\frac{99}{2} \sin t-\frac{19}{54} \sin (3 t) \\
& q_{3}(t)=-\frac{43}{12} \cos ^{2} t \cos (3 t)-\frac{3914}{625} \cos ^{5} t+\frac{9701909}{40500} \cos ^{3} t+\frac{26472442}{3375} \cos t \\
& q_{4}(t)=\frac{19}{6} \cos ^{2} t \cos (3 t)+\frac{23936}{625} \cos ^{5} t-\frac{31274533}{20250} \cos ^{3} t-\frac{216658108}{3375} \cos t \\
& q_{5}(t)=\frac{236}{25} \cos ^{5} t+\frac{200564}{405} \cos ^{3} t+\frac{3396328}{135} \cos t \\
& q_{6}(t)=-\frac{129}{4} \cos ^{4} t \sin (3 t)-\frac{1849}{432} \cos t \cos ^{2}(3 t)-\frac{43}{2} \cos ^{3} t \sin t \cos (3 t)+\frac{1075}{8} \cos ^{2} t \cos (3 t) \\
& -\frac{11742}{125} \cos ^{6} t \sin t+\frac{9701909}{4500} \cos ^{4} t \sin t+\frac{26472442}{1125} \cos ^{2} t \sin t-\frac{16875}{16} \cos ^{3} t \\
& q_{7}(t)=\frac{57}{2} \cos ^{4} t \sin (3 t)+\frac{817}{108} \cos t \cos ^{2}(3 t)+19 \cos ^{3} t \sin t \cos (3 t)-\frac{947}{2} \cos ^{2} t \cos (3 t) \\
& +\frac{71808}{125} \cos ^{6} t \sin t-\frac{31274533}{2250} \cos ^{4} t \sin t-\frac{216658108}{1125} \cos ^{2} t \sin t+\frac{22275}{4} \cos ^{3} t \\
& q_{8}(t)=-\frac{361}{108} \cos t \cos ^{2}(3 t)+\frac{627}{2} \cos ^{2} t \cos (3 t)-\frac{708}{5} \cos ^{6} t \sin t \frac{200564}{45} \cos ^{4} t \sin t \\
& +\frac{3396328}{45} \cos ^{2} t \sin t-\frac{29403}{4} \cos ^{3} t \\
& q_{9}(t)=\frac{285 \sin (7 t)}{4802}+\frac{52079 \cos (7 t)}{172872}+\frac{57 \sin (5 t)}{125}+\frac{8683 \cos (5 t)}{9000}-\frac{87932299816 \sin ^{5} t}{16078125} \\
& +\frac{169628431490024 \sin ^{3} t}{260465625}-\frac{2154223646101879 \sin t}{173643750}-\frac{158316 \cos ^{5} t}{125} \\
& +\frac{51050891 \cos ^{3} t}{1350}+\frac{1911052939 \cos t}{900}
\end{aligned}
$$




\section{The Adomian Decomposition Method}

\subsection{Generalities}

General properties of ADM and its application can be found in (ABBAOUI, K., 1995; ABBAOUI, K., \& CHERRUAULT, Y., 1994; ABBAOUI, K., \& CHERRUAULT, Y., 1999; NGARHASTA, N. \& et al., 2002). Some of these are outlined as follows. Suppose that we need to solve the following equation

$$
A u=f
$$

in a real Hilbert space $H$, where $A: H \rightarrow H$ is a linear or a nonlinear operator, $f \in H$ are given; and $u \in H$ is the unknown. The principle of the ADM is based on the decomposition of the nonlinear operator $A$ in the following form:

$$
A=L+R+N
$$

where $L+R$ is linear, $N$ nonlinear, $L$ invertible with $L^{-1}$ as inverse. Using that decomposition, equation (21) is equivalent to

$$
u=\theta+L^{-1} f-L^{-1} R u-L^{-1} N u
$$

where $\theta$ satisfies $L \theta=0$. Equation (22) is called the Adomian fundamental equation or Adomian canonical form. We look for the solution of (21) in a series expansion form $u=\sum_{n=0}^{+\infty} u_{n}$ and we consider $N u=\sum_{n=0}^{+\infty} A_{n}$ where $A_{n}$ are special polynomials of variables $u_{0}, u_{1}, \ldots, u_{n}$ called Adomian polynomials and defined by (ABBAOUI, K., 1995; ABBAOUI, K., \& CHERRUAULT, Y., 1994; ABBAOUI, K., \& CHERRUAULT, Y., 1999; NGARHASTA, N. \& et al., 2002):

$$
A_{n}=\frac{1}{n !} \frac{d^{n}}{d \lambda^{n}}\left[N\left(\sum_{i=0}^{+\infty} \lambda^{i} u_{i}\right)\right]_{\lambda=0} \quad n=0,1,2, \ldots
$$

where $\lambda$ is a parameter used by "convenience". Thus (22) can be rewritten as follows :

$$
\sum_{n=0}^{+\infty} u_{n}=\theta+L^{-1} f-L^{-1} R\left(\sum_{n=0}^{+\infty} u_{n}\right)-L^{-1}\left(\sum_{n=0}^{+\infty} A_{n}\right) .
$$

We suppose that the series $\sum_{n=0}^{+\infty} u_{n}$ and $\sum_{n=0}^{+\infty} A_{n}$ are convergent, and obtained by identification the Adomian algorithm :

$$
\left\{\begin{array}{c}
u_{0}=\theta+L^{-1} f \\
u_{1}=-L^{-1}\left(R u_{0}\right)-L^{-1} A_{0} \\
\cdot \\
\cdot \\
u_{n+1}=-L^{-1}\left(R u_{n}\right)-L^{-1} A_{n}
\end{array} .\right.
$$

In practice it is often difficult to calculate all the terms of an Adomian series; so we approach the series solution by the truncated series $u=\sum_{i=0}^{n} u_{i}$, where the choice of $n$ depends on error requirements.

\subsection{Application of ADM}

Defining the operators :

$$
L_{t}(\bullet)=\frac{\partial^{2}}{\partial t^{2}}(\bullet), \quad L_{x}(\bullet)=\frac{\partial^{2}}{\partial x^{2}}(\bullet), \quad N u=\left(\frac{\partial u}{\partial t}\right)^{3} \quad \text { and } \quad L_{t}^{-1}(\bullet)=\int_{0}^{t} \int_{0}^{z}(\bullet) d s d z
$$

Equation (1) can be written as :

$$
L_{t} u-L_{x} u=-\varepsilon N u
$$

Applying $L_{t}^{-1}$ to (24), we obtain :

$$
u(t, x)=u(0, x)+t \frac{\partial u}{\partial t}(0, x)+L_{t}^{-1}\left[L_{x} u(t, x)\right]-\varepsilon L_{t}^{-1}[N u(t, x)]
$$


Assuming that the solution of (1) can be given by :

$$
u(t, x)=\sum_{n=0}^{+\infty} u_{n}(t, x)
$$

and

$$
N u(t, x)=\sum_{n=0}^{+\infty} A_{n}(t, x)
$$

where $A_{n}$ are the Adomian's polynomials with

$$
A_{n}=\frac{1}{n !}\left[\frac{d^{n}}{d \lambda^{n}}\left(N\left(\sum_{i=0}^{+\infty} \lambda^{i} u_{i}\right)\right)\right]_{\lambda=0}
$$

By substituting (26) and (27) into (25), we obtain the Adomian algorithm

$$
\left\{\begin{array}{c}
u_{0}(t, x)=u(0, x)+t \frac{\partial u}{\partial t}(0, x)=\cos x \\
u_{n+1}(t, x)=L_{t}^{-1}\left[L_{x} u_{n}(t, x)\right]-\varepsilon L_{t}^{-1}\left[A_{n}(t, x)\right], \quad n \geq 0
\end{array}\right.
$$

with

$$
\begin{aligned}
A_{0}= & \left(\frac{\partial u_{0}}{\partial t}\right)^{3} \\
A_{1}= & 3 \varepsilon\left(\frac{\partial u_{0}}{\partial t}\right)^{2}\left(\frac{\partial^{2} u_{1}}{\partial t}\right) \\
A_{2}= & 3 \varepsilon\left(\frac{\partial u_{0}}{\partial t}\right)^{2}\left(\frac{\partial u_{2}}{\partial t}\right)+3 \varepsilon\left(\frac{\partial u_{0}}{\partial t}\right)\left(\frac{\partial u_{1}}{\partial t}\right)^{2} \\
A_{3}= & 3 \varepsilon\left(\frac{\partial u_{0}}{\partial t}\right)^{2}\left(\frac{\partial u_{3}}{\partial t}\right)+6 \varepsilon\left(\frac{\partial u_{0}}{\partial t} \frac{\partial u_{1}}{\partial t} \frac{\partial u_{2}}{\partial t}\right)+\varepsilon\left(\frac{\partial u_{1}}{\partial t}\right)^{3} \\
A_{4}= & 3 \varepsilon\left(\frac{\partial u_{0}}{\partial t}\right)^{2}\left(\frac{\partial u_{4}}{\partial t}\right)+6 \varepsilon\left(\frac{\partial u_{0}}{\partial t} \frac{\partial u_{1}}{\partial t} \frac{\partial u_{3}}{\partial t}\right)+3 \varepsilon\left(\frac{\partial u_{0}}{\partial t}\right)\left(\frac{\partial u_{2}}{\partial t}\right)^{2}+3 \varepsilon\left(\frac{\partial u_{1}}{\partial t}\right)^{2}\left(\frac{\partial u_{2}}{\partial t}\right) \\
A_{5}= & 3 \varepsilon\left(\frac{\partial u_{0}}{\partial t}\right)^{2}\left(\frac{\partial u_{5}}{\partial t}\right)+6 \varepsilon\left(\frac{\partial u_{0}}{\partial t} \frac{\partial u_{1}}{\partial t} \frac{\partial u_{4}}{\partial t}\right)+6 \varepsilon\left(\frac{\partial u_{0}}{\partial t} \frac{\partial u_{2}}{\partial t} \frac{\partial u_{3}}{\partial t}\right)+3 \varepsilon\left(\frac{\partial u_{1}}{\partial t}\right)^{2}\left(\frac{\partial u_{3}}{\partial t}\right) \\
& \left.+3 \varepsilon\left(\frac{\partial u_{1}}{\partial t}\right)\left(\frac{\partial u_{2}}{\partial t}\right)^{2}\right)
\end{aligned}
$$

Using the algorithm, we have :

$$
\begin{aligned}
& u_{0}(t, x)=\cos x \\
& u_{1}(t, x)=-\frac{1}{2} t^{2} \cos x \\
& u_{2}(t, x)=\frac{1}{24} t^{4} \cos x \\
& u_{3}(t, x)=-\frac{1}{720} t^{6} \cos x+\frac{\varepsilon}{20} t^{5} \cos ^{3} x \\
& u_{4}(t, x)=\frac{\varepsilon}{140} t^{7} \cos x \sin ^{2} x-\frac{13 \varepsilon}{840} t^{7} \cos ^{3} x+\frac{1}{40320} t^{8} \cos x \\
& u_{5}(t, x)=-\frac{1}{3628800} t^{10} \cos x-\frac{\varepsilon}{504} t^{9} \cos x \sin ^{2} x+\frac{71 \varepsilon}{30240} t^{9} \cos ^{3} x-\frac{3 \varepsilon^{2}}{224} t^{8} \cos ^{5} x
\end{aligned}
$$


Finally, the approximate solution of (1) is given by :

$$
\begin{aligned}
& u(t, x) \simeq u_{0}(t, x)+u_{1}(t, x)+u_{2}(t, x)+\cdots+u_{10}(t, x) \\
& \simeq\left[\cos x\left(1-\frac{t^{2}}{2 !}+\frac{t^{4}}{4 !}-\frac{t^{6}}{6 !}+\frac{t^{8}}{8 !}-\frac{t^{10}}{10 !}+\frac{t^{12}}{12 !}-\frac{t^{14}}{14 !}+\frac{t^{16}}{16 !}-\frac{t^{18}}{18 !}+\frac{t^{20}}{20 !}\right)\right]+ \\
& \varepsilon\left[\operatorname { c o s } ^ { 3 } x \left(\frac{t^{5}}{20}-\frac{13 t^{7}}{840}+\frac{71 t^{9}}{30240}-\frac{491 t^{11}}{3326400}+\frac{13711 t^{13}}{1037836800}-\frac{28607 t^{15}}{43589145600}\right.\right. \\
& +\frac{66811 t^{17}}{2694601728000}-\frac{7198319 t^{19}}{10137091700736000}+\frac{t^{21}}{13502619648000} \\
& \left.-\frac{61 t^{23}}{47826278793216000}+\frac{547 t^{25}}{28695767275929600000}\right) \\
& +\cos x \sin ^{2} x\left(\frac{t^{7}}{140}-\frac{t^{9}}{504}+\frac{47 t^{11}}{184800}-\frac{739 t^{13}}{43243200}+\frac{34403 t^{15}}{36324288000}-\frac{727 t^{17}}{18712512000}\right. \\
& \left.\left.+\frac{188947 t^{19}}{153592298496000}-\frac{t^{21}}{4725916876800}+\frac{13 t^{23}}{3416162770944000}-\frac{41 t^{25}}{717394181898240000}\right)\right] \\
& +\varepsilon^{2}\left[\operatorname { c o s } ^ { 5 } x \left(-\frac{3 t^{8}}{224}+\frac{439 t^{10}}{100800}-\frac{23801 t^{12}}{13305600}+\frac{743201 t^{14}}{2421619200}-\frac{38239 t^{16}}{931392000}+\frac{78121361 t^{18}}{22230464256000}\right.\right. \\
& \left.-\frac{1903 t^{20}}{482236416000}+\frac{5921 t^{22}}{31191051386880}-\frac{297239 t^{24}}{31304473391923200}\right) \\
& +\cos ^{3} x \sin ^{2} x\left(-\frac{13 t^{10}}{2800}+\frac{3397 t^{12}}{1663200}-\frac{352421 t^{14}}{605404800}+\frac{83371 t^{16}}{825552000}-\frac{26118227 t^{18}}{2223046425600}\right. \\
& \left.+\frac{1247 t^{20}}{42195686400}-\frac{1191041 t^{22}}{779776284672000}+\frac{11675131 t^{24}}{215218254569472000}+\frac{12517 t^{25}}{1016715110400000}\right) \\
& +\cos x \sin ^{4} x\left(-\frac{13 t^{12}}{61600}+\frac{1663 t^{14}}{20180160}-\frac{16447 t^{16}}{864864000}+\frac{573889 t^{18}}{205837632000}-\frac{2903 t^{20}}{281304576000}\right. \\
& \left.\left.+\frac{12517 t^{23}}{20334302208000}-\frac{1191041 t^{24}}{71739418189824000}-\frac{162721 t^{25}}{12200581324800000}\right)\right]+ \\
& \varepsilon^{3}\left[\operatorname { c o s } ^ { 7 } x \left(\frac{57 t^{11}}{12320}-\frac{1319 t^{13}}{443520}+\frac{54919 t^{14}}{47297250}+\frac{83, t^{15}}{470400}-\frac{54919 t^{16}}{1621620000}-\frac{227253749 t^{17}}{823350528000}\right.\right. \\
& \left.+\frac{1901771 t^{19}}{1969132032000}-\frac{37259 t^{21}}{170031960000}+\frac{215576267 t^{23}}{7608726171648000}\right)+\cos ^{5} x \sin ^{2} x\left(\frac{839 t^{13}}{320320}\right. \\
& \left.-\frac{165827 t^{15}}{84084000}+\frac{54919 t^{16}}{270270000}+\frac{76842539 t^{17}}{137225088000}-\frac{947 t^{19}}{202585600}+\frac{5031053 t^{21}}{7254696960000}-\frac{34493909 t^{23}}{333716060160000}\right) \\
& +\cos ^{3} x \sin ^{4} x\left(+\frac{193 t^{15}}{600600}-\frac{65171 t^{17}}{285885600}+\frac{129 t^{19}}{40517120}-\frac{5819 t^{21}}{12155136000}+\frac{277663 t^{23}}{4359166035840}\right)+ \\
& \left.\cos x \sin ^{6} x\left(\frac{193 t^{17}}{27227200}-\frac{t^{19}}{6077568}+\frac{193 t^{21}}{3646540800}-\frac{35353 t^{23}}{4612874112000}\right)\right] \\
& +\cdots
\end{aligned}
$$

This solution can be written in the form :

$$
\begin{gathered}
u(t, x) \simeq \quad \cos t \cos x+\varepsilon\left[l_{1}(t) \cos ^{3} x+l_{2}(t) \cos x \sin ^{2} x\right] \\
+\varepsilon^{2}\left[l_{3}(t) \cos ^{5} x+l_{4}(t) \cos ^{3} x \sin ^{2} x+l_{5}(t) \cos x \sin ^{4} x\right] \\
+\varepsilon^{3}\left[l_{6}(t) \cos ^{7} x+l_{7}(t) \cos ^{5} x \sin ^{2} x+l_{8}(t) \cos ^{3} x \sin ^{4} x+l_{9}(t) \cos x \sin ^{6} x\right] \\
+\cdots \\
l_{1}(t)=\frac{t^{5}}{20}-\frac{13 t^{7}}{840}+\frac{71 t^{9}}{30240}-\frac{491 t^{11}}{3326400}+\frac{13711 t^{13}}{1037836800}-\frac{28607 t^{15}}{43589145600}+\frac{66811 t^{17}}{2694601728000} \\
-\frac{7198319 t^{19}}{10137091700736000}+\frac{t^{21}}{13502619648000}-\frac{547 t^{25}}{47826278793216000}+\frac{5695767275929600000}{286957}
\end{gathered}
$$




$$
\begin{aligned}
l_{2}(t)= & \frac{t^{7}}{140}-\frac{t^{9}}{504}+\frac{47 t^{11}}{184800}-\frac{739 t^{13}}{43243200}+\frac{34403 t^{15}}{36324288000}-\frac{727 t^{17}}{18712512000}+\frac{188947 t^{19}}{153592298496000} \\
& -\frac{t^{21}}{4725916876800}+\frac{13 t^{23}}{3416162770944000}-\frac{41 t^{25}}{717394181898240000} \\
l_{3}(t)= & -\frac{3 t^{8}}{224}+\frac{439 t^{10}}{100800}-\frac{23801 t^{12}}{13305600}+\frac{743201 t^{14}}{2421619200}-\frac{38239 t^{16}}{931392000}+\frac{78121361 t^{18}}{22230464256000}-\frac{1903 t^{20}}{482236416000} \\
& +\frac{5921 t^{22}}{31191051386880}-\frac{297239 t^{24}}{31304473391923200}
\end{aligned}
$$$$
l_{4}(t)=-\frac{13 t^{10}}{2800}+\frac{3397 t^{12}}{1663200}-\frac{352421 t^{14}}{605404800}+\frac{83371 t^{16}}{825552000}-\frac{26118227 t^{18}}{2223046425600}+\frac{1247 t^{20}}{42195686400}
$$$$
-\frac{1191041 t^{22}}{779776284672000}+\frac{11675131 t^{24}}{215218254569472000}+\frac{12517 t^{25}}{1016715110400000}
$$

$$
\begin{aligned}
l_{5}(t)= & -\frac{13 t^{12}}{61600}+\frac{1663 t^{14}}{20180160}-\frac{16447 t^{16}}{864864000}+\frac{573889 t^{18}}{205837632000}-\frac{2903 t^{20}}{281304576000}+\frac{12517 t^{23}}{20334302208000} \\
& -\frac{1191041 t^{24}}{71739418189824000}-\frac{162721 t^{25}}{12200581324800000}
\end{aligned}
$$

$$
\begin{aligned}
l_{6}(t)= & \frac{57 t^{11}}{12320}-\frac{1319 t^{13}}{443520}+\frac{54919 t^{14}}{47297250}+\frac{83, t^{15}}{470400}-\frac{54919 t^{16}}{1621620000}-\frac{227253749 t^{17}}{823350528000}+\frac{1901771 t^{19}}{1969132032000} \\
& -\frac{37259 t^{21}}{170031960000}+\frac{215576267 t^{23}}{7608726171648000}
\end{aligned}
$$

$$
\begin{aligned}
l_{7}(t)= & \frac{839 t^{13}}{320320}-\frac{165827 t^{15}}{84084000}+\frac{54919 t^{16}}{270270000}+\frac{76842539 t^{17}}{137225088000}-\frac{947 t^{19}}{202585600}+\frac{5031053 t^{21}}{7254696960000} \\
& -\frac{34493909 t^{23}}{333716060160000}
\end{aligned}
$$

$$
\begin{aligned}
& l_{8}(t)=\frac{193 t^{15}}{600600}-\frac{65171 t^{17}}{285885600}+\frac{129 t^{19}}{40517120}-\frac{5819 t^{21}}{12155136000}+\frac{277663 t^{23}}{4359166035840} \\
& l_{9}(t)=\frac{193 t^{17}}{27227200}-\frac{t^{19}}{6077568}+\frac{193 t^{21}}{3646540800}-\frac{35353 t^{23}}{4612874112000}
\end{aligned}
$$

\section{Comparison of the Approximate Solutions}

In this section we analyze the approximate solutions of (1) are obtained by the three numerical methods (ADM, RPM and ADM).

The solution in equation (1) which obtained by HPM is absolutely same as that of the solution obtained by ADM. Furthermore, the main advantage in using the HPM for solving the considered model is that the approximate solutions obtained successfully without requiring a small parameter in the equation and without calculating the complicated Adomian's polynomials. The approximate solution obtained by RPM, differs from the one obtained by ADM and HPM.

The tables (1), (3) and (5) give some values of the approximate solutions obtained by the three methods. One notes a 
significant variation of the values obtained by RPM when $\varepsilon$ becomes increasingly large.

The tables (2), (4) and (6) show absolute error between the various approximate solutions. It is noticed that the absolute error between the solutions of RPM and ADM just as RPM and HPM increases when $\varepsilon$ becomes increasingly large.

Table 1. Approximate solutions by ADM, RPM and HPM, for $t=0.2$ and $\varepsilon=0.001$

\begin{tabular}{|cccc|}
\hline$x$ & $u_{\text {adm }}$ & $u_{\text {rpm }}$ & $u_{h p m}$ \\
\hline 0 & 0.9801 & 0.9850 & 0.9801 \\
\hline 0.1 & 0.9752 & 0.9793 & 0.9752 \\
\hline 0.2 & 0.9605 & 0.9626 & 0.9605 \\
\hline 0.3 & 0.9363 & 0.9355 & 0.9363 \\
\hline 0.4 & 0.9027 & 0.8992 & 0.9027 \\
\hline 0.5 & 0.8601 & 0.8546 & 0.8601 \\
\hline 0.6 & 0.8089 & 0.8027 & 0.8089 \\
\hline 0.7 & 0.7496 & 0.7443 & 0.7496 \\
\hline 0.8 & 0.6828 & 0.6798 & 0.6828 \\
\hline 0.9 & 0.6092 & 0.6093 & 0.6092 \\
\hline 1 & 0.5295 & 0.5328 & 0.5295 \\
\hline
\end{tabular}

Table 2. Absolute error for variables $x$ from 0 to 1 and $t=0.2$ and $\varepsilon=0.001$

\begin{tabular}{|cccc|}
\hline$x$ & $\left|u_{\text {adm }}-u_{r p m}\right|$ & $\left|u_{a d m}-u_{h p m}\right|$ & $\left|u_{h p m}-u_{r p m}\right|$ \\
\hline 0 & 0.0049 & 0 & 0.0049 \\
\hline 0.1 & 0.0041 & 0 & 0.0041 \\
\hline 0.2 & 0.0021 & 0 & 0.0021 \\
\hline 0.3 & 0.0008 & 0 & 0.0008 \\
\hline 0.4 & 0.0035 & 0 & 0.0035 \\
\hline 0.5 & 0.0055 & 0 & 0.0055 \\
\hline 0.6 & 0.0062 & 0 & 0.0062 \\
\hline 0.7 & 0.0053 & 0 & 0.0053 \\
\hline 0.8 & 0.0030 & 0 & 0.0030 \\
\hline 0.9 & 0.0001 & 0 & 0.0001 \\
\hline 1 & 0.0033 & 0 & 0.0033 \\
\hline
\end{tabular}

Table 3. Approximate solutions by ADM, RPM and HPM, for $t=0.5$ and $\varepsilon=0.002$

\begin{tabular}{|cccc|}
\hline$x$ & $u_{\text {adm }}$ & $u_{\text {rpm }}$ & $u_{\text {hpm }}$ \\
\hline 0 & 0.8776 & 0.9040 & 0.8776 \\
\hline 0.1 & 0.8732 & 0.8946 & 0.8732 \\
\hline 0.2 & 0.8601 & 0.8681 & 0.8601 \\
\hline 0.3 & 0.8384 & 0.8681 & 0.8384 \\
\hline 0.4 & 0.8083 & 0.7843 & 0.8083 \\
\hline 0.5 & 0.7702 & 0.7382 & 0.7702 \\
\hline 0.6 & 0.7243 & 0.6936 & 0.7243 \\
\hline 0.7 & 0.6712 & 0.6505 & 0.6712 \\
\hline 0.8 & 0.6114 & 0.6058 & 0.6114 \\
\hline 0.9 & 0.5455 & 0.5558 & 0.5455 \\
\hline 1 & 0.4742 & 0.4969 & 0.4742 \\
\hline
\end{tabular}


Table 4. Absolute error for variables $x$ from 0 to 1 and $t=0.5$ and $\varepsilon=0.002$

\begin{tabular}{|cccc|}
\hline$x$ & $\left|u_{\text {adm }}-u_{\text {rpm }}\right|$ & $\left|u_{\text {adm }}-u_{h p m}\right|$ & $\left|u_{h p m}-u_{\text {rpm }}\right|$ \\
\hline 0 & 0.0264 & 0 & 0.0264 \\
\hline 0.1 & 0.0214 & 0 & 0.0214 \\
\hline 0.2 & 0.0080 & 0 & 0.0080 \\
\hline 0.3 & 0.0090 & 0 & 0.0090 \\
\hline 0.4 & 0.0240 & 0 & 0.0240 \\
\hline 0.5 & 0.0320 & 0 & 0.0320 \\
\hline 0.6 & 0.0307 & 0 & 0.0307 \\
\hline 0.7 & 0.0207 & 0 & 0.0207 \\
\hline 0.8 & 0.0056 & 0 & 0.0056 \\
\hline 0.9 & 0.0103 & 0 & 0.0103 \\
\hline 1 & 0.0227 & 0 & 0.0227 \\
\hline
\end{tabular}

Table 5. Approximate solutions by ADM, RPM and HPM, for $t=0.8$ and $\varepsilon=0.003$

\begin{tabular}{|cccc|}
\hline$x$ & $u_{\text {adm }}$ & $u_{\text {rpm }}$ & $u_{\text {hpm }}$ \\
\hline 0 & 0.6967 & 0.7921 & 0.6967 \\
\hline 0.1 & 0.6933 & 0.7694 & 0.6933 \\
\hline 0.2 & 0.6933 & 0.7086 & 0.6933 \\
\hline 0.3 & 0.6656 & 0.6293 & 0.6656 \\
\hline 0.4 & 0.6417 & 0.5550 & 0.6417 \\
\hline 0.5 & 0.6114 & 0.5042 & 0.6114 \\
\hline 0.6 & 0.5750 & 0.4831 & 0.5750 \\
\hline 0.7 & 0.5329 & 0.4844 & 0.5329 \\
\hline 0.8 & 0.4854 & 0.4913 & 0.4854 \\
\hline 0.9 & 0.4331 & 0.4854 & 0.4331 \\
\hline 1 & 0.3764 & 0.4537 & 0.3764 \\
\hline
\end{tabular}

Table 6. Absolute error for variables $x$ from 0 to 1 and $t=0.8$ and $\varepsilon=0.003$

\begin{tabular}{|cccc|}
\hline$x$ & $\left|u_{\text {adm }}-u_{\text {rpm }}\right|$ & $\left|u_{\text {adm }}-u_{h p m}\right|$ & $\left|u_{h p m}-u_{\text {rpm }}\right|$ \\
\hline 0 & 0.0954 & 0 & 0.0954 \\
\hline 0.1 & 0.0761 & 0 & 0.0761 \\
\hline 0.2 & 0.0257 & 0 & 0.0257 \\
\hline 0.3 & 0.0363 & 0 & 0.0363 \\
\hline 0.4 & 0.0867 & 0 & 0.0867 \\
\hline 0.5 & 0.1072 & 0 & 0.1072 \\
\hline 0.6 & 0.0919 & 0 & 0.0919 \\
\hline 0.7 & 0.0485 & 0 & 0.0485 \\
\hline 0.8 & 0.0059 & 0 & 0.0059 \\
\hline 0.9 & 0.0523 & 0 & 0.0523 \\
\hline 1 & 0.0773 & 0 & 0.0773 \\
\hline
\end{tabular}

The figures (1) and (2) give the comparison of the approximate solutions in dimension 2, obtained by the three methods. In dimension 3, we obtain the figures (3), (4),(5) and (6). 


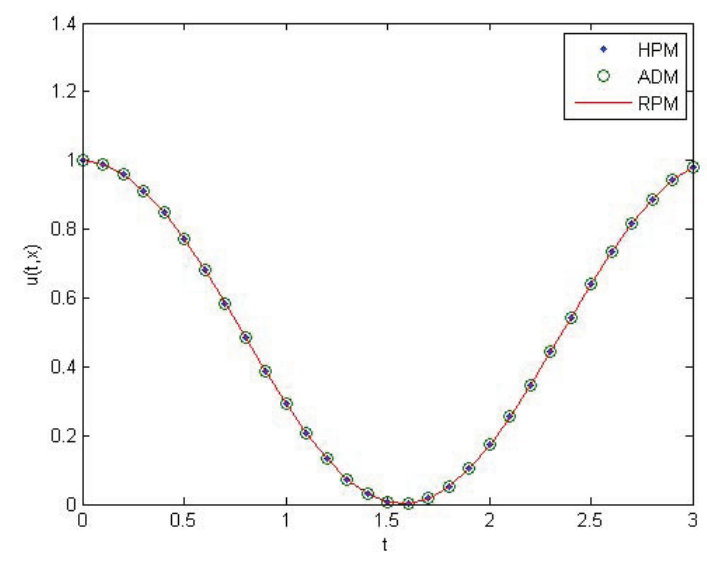

(a) $\varepsilon=0.0001$

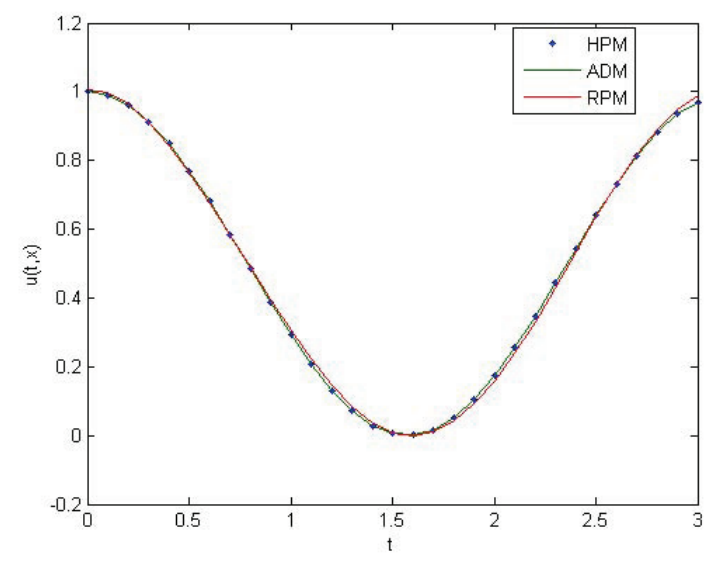

(b) $\varepsilon=0.001$

Figure 1. Comparison of the HPM solution, ADM solution and RPM solution

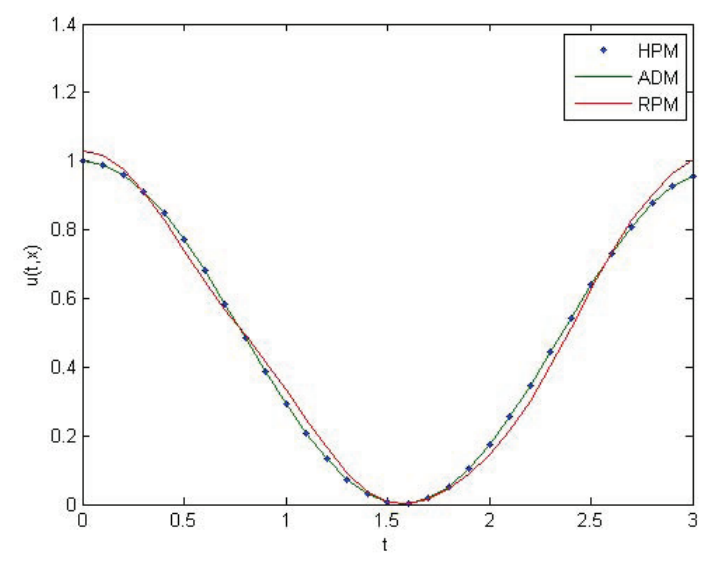

(a) $\varepsilon=0.002$

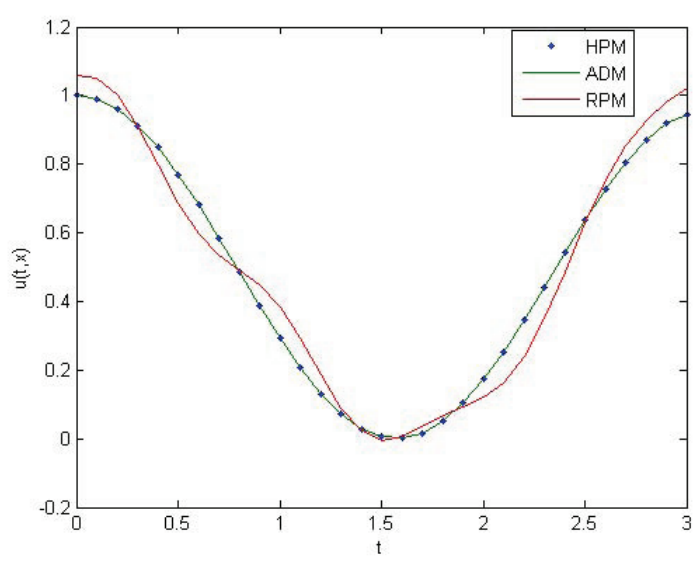

(b) $\varepsilon=0.003$

Figure 2. Comparison of the HPM solution, ADM solution and RPM solution

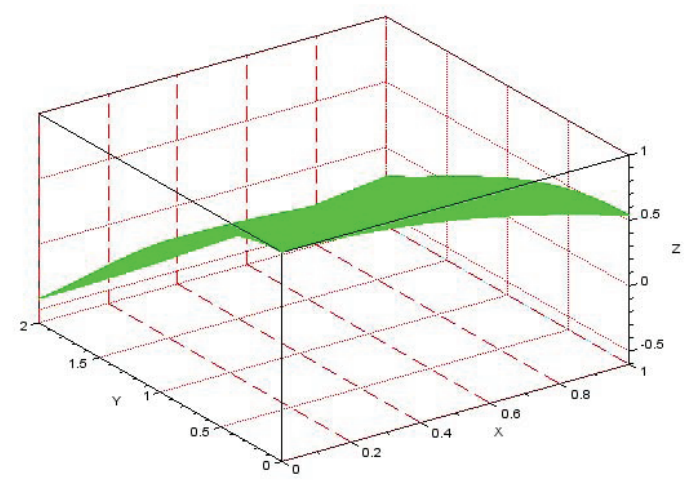

(a) HPM solution

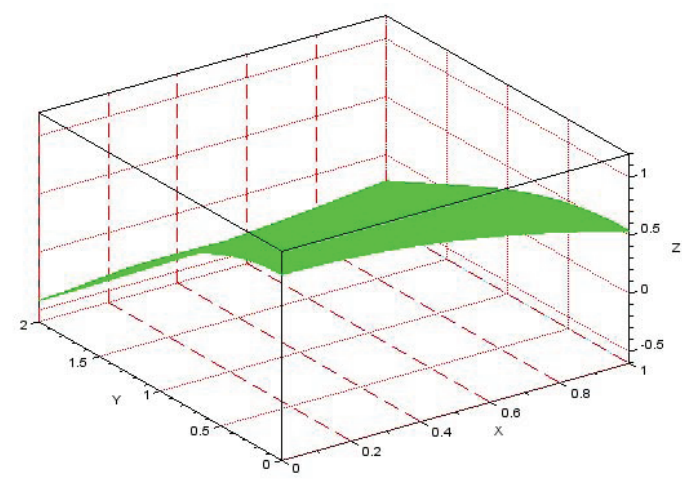

(b) RPM solution

Figure 3. Comparison of the HPM solution with RPM solution for $\varepsilon=0.0001$ 


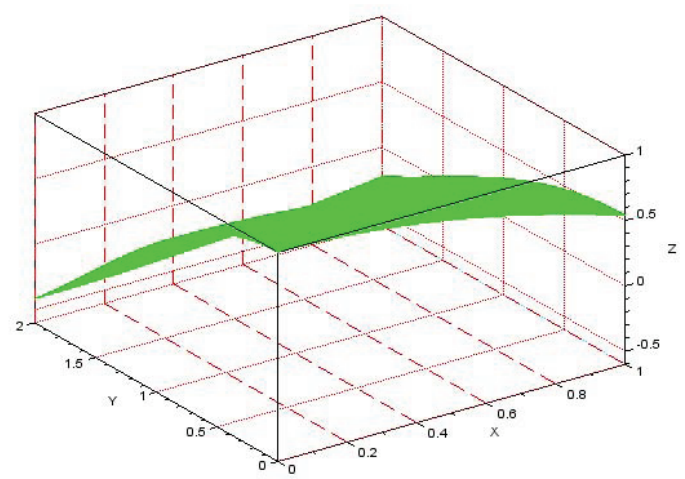

(a) ADM solution

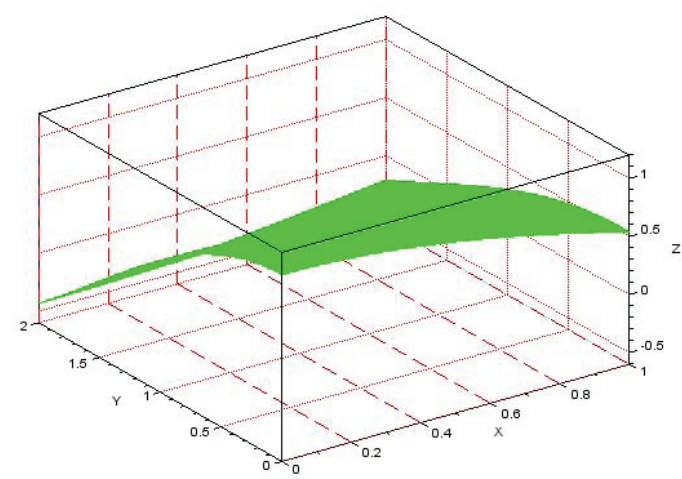

(b) RPM solution

Figure 4. Comparison of the HPM solution with RPM solution for $\varepsilon=0.001$

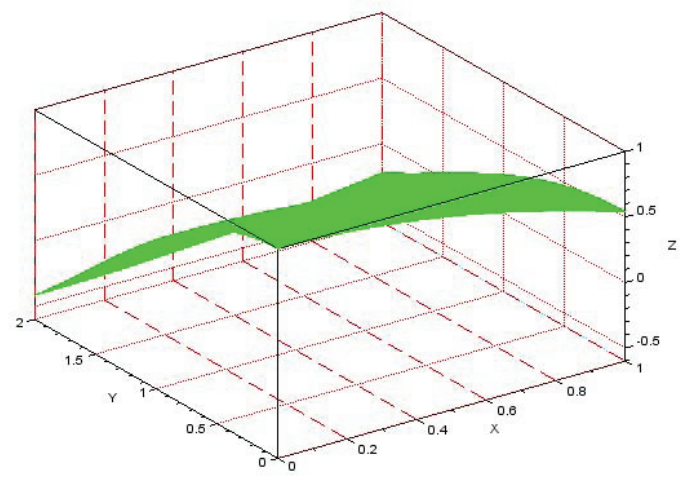

(a) HPM solution

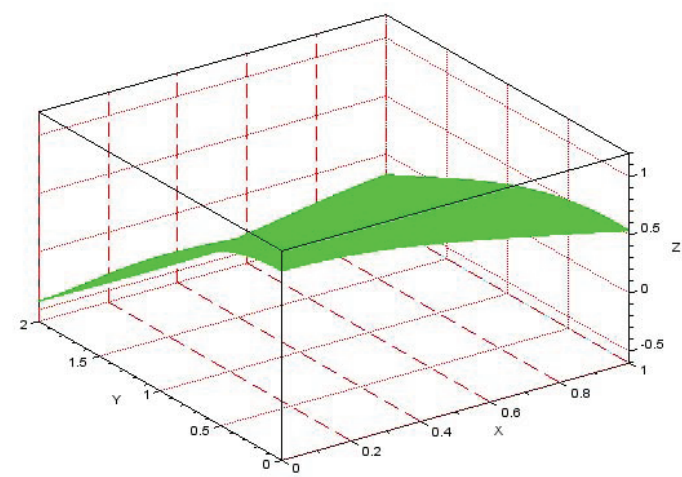

(b) RPM solution

Figure 5. Comparison of the HPM solution with RPM solution for $\varepsilon=0.002$

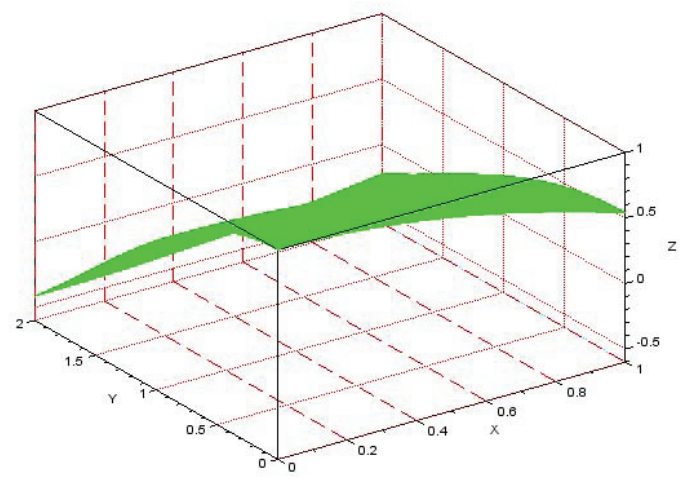

(a) ADM solution

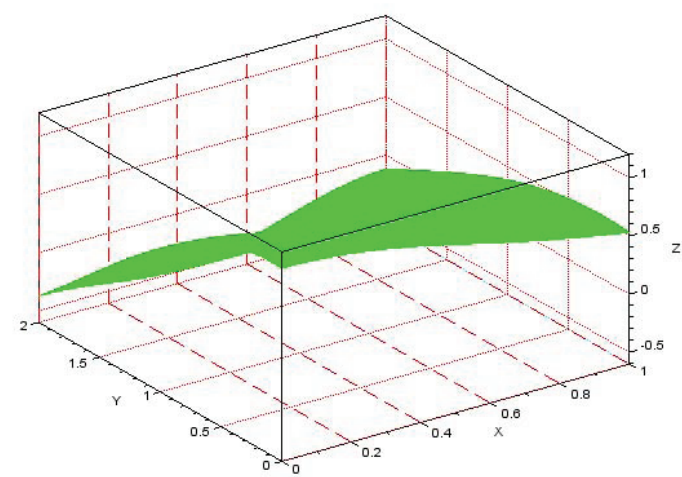

(b) RPM solution

Figure 6. Comparison of the HPM solution with RPM solution for $\varepsilon=0.003$ 


\section{Conclusion}

In this paper, the HPM, ADM and RPM have been successfully employed to obtain the approximate analytical solutions of the wave equation with cubic damping. Finally, the results obtained by three methods were comapared. RPM solution differs from the ADM and HPM solution, but ADM and HPM give the same approximate solution. This research reveals that although the obtained results by HPM and ADM are the same, HPM are much easier, more convenient, and efficient in comparison. Different from ADM, where specific algorithms are usually used to determine the Adomian polynomials, HPM handle linear and nonlinear problem in simple manner by deforming a difficult problem into a simple one.

\section{References}

ABBAOUI, K. (1995). Les fondements de la méthode décompositionnelle d'Adomian et application à la résolution de problèmes issus de la biologie et de la médécine. Thèse de doctorat de l’Université Paris VI. Octobre.

ABBAOUI, K., \& CHERRUAULT, Y. (1994). Convergence of Adomian method applied to non linear equations. Math. Comput. Modelling, 20(9), 60-73. https://doi.org/10.1016/0895-7177(94)00163-4

ABBAOUI, K., \& CHERRUAULT, Y. (1999). The Decomposition method applied to the Cauchy problem. Kybernetes, 28(1), 68-74. https://doi.org/10.1108/03684929910253261

NGARHASTA, N., SOME, B., ABBAOUI, K., \& CHERRUAULT, Y. (2002). New numerical study of Adomian method applied to a diffusion model. Kybernetes, 31(1), 61-75. https://doi.org/10.1108/03684920210413764

Oke, M. O. (2015). On Adomian Decomposition Method for solving general wave equations on transmission lines. European Journal of Basic and Applied Sciences, 2(2), ISSN 2059-3058.

Ghoreishi, M., Ismail, A. I. B. Md., \& Ali, N. H. M. (2010). Adomian Decomposition Method (ADM) for Nonlinear Wave-like Equations with Variable Coefficient. Applied Mathematical Sciences, 4(49), 2431-2444.

He, J. H. (1999). Homotopy perturbation technique. Computer Methods in Applied Mechanics and Engineering, 178, 257-262.

He, J. H. (2000). A coupling method of homotopy technique and perturbation technique for nonlinear problems, Int J Non-Linear Mech, 35, 37-43.

He, J. H. (2003). Homotopy perturbation method : a new nonlinear analytical technique Applied Mathematics and Computation, 135, 73-79.

He, J. H. (2004). The homotopy perturbation method for nonlinear oscillators with discontinuities. Applied Mathematics and Computation, 151(1), 287-292.

Gupta, S., Kumar, D., \& Singh, J. (2013). Application of He's homotopy perturbation method for solving nonlinear wavelike equations with variable coefficients. International Journal of Advances in Applied Mathematics and Mechanics, 1(2), 65-79. ISSN: 2347-2529.

Ghazanfari, B., Ghazanfari, A. G., \& Fuladvand, M. (2011). Modification of the Homotopy Perturbation Method for Numerical Solution of Nonlinear Wave and System of Nonlinear Wave Equations. The Journal of Mathematics and Computer Science, 3(2), 212-224.

JAGER, DE. E. M., \& JIANG, FU RU, (1996). The theory of singular perturbations. North-Holland series in Applied Mathematics and Mechanics, 42.

\section{Copyrights}

Copyright for this article is retained by the author(s), with first publication rights granted to the journal.

This is an open-access article distributed under the terms and conditions of the Creative Commons Attribution license (http://creativecommons.org/licenses/by/4.0/). 